\title{
Extension of the random phase approximation including the self-consistent coupling to two-phonon contributions
}

\author{
C. Barbieri* \\ TRIUMF, 4004 Wesbrook Mall, Vancouver, British Columbia, Canada V6T 2A3 \\ W. H. Dickhoff \\ Department of Physics, Washington University, St. Louis, Missouri 63130, USA
}

(Received 4 December 2002; published 29 July 2003)

\begin{abstract}
A microscopic formalism is developed that includes the coupling to two particle-hole phonons in the particle-hole propagator by extending the dressed random phase approximation (DRPA) equation for a finite system. The resulting formalism is applied to study the low-lying excitation spectrum of ${ }^{16} \mathrm{O}$. It is observed that the coupling to two-phonon states at low energy generates excited states with quantum numbers that cannot be obtained in the DRPA approach. Nevertheless, the two-phonon states mix weakly with particle-hole configurations and participate only partially in the formation of the lowest-lying positive-parity excited states. The stability of the present calculation is tested vs the truncation of model space. It is demonstrated that when single-particle strength fragmentation is properly considered, the present formalism exhibits convergence with respect to the chosen model space within the confines of the chosen approximation scheme.
\end{abstract}

DOI: 10.1103/PhysRevC.68.014311

PACS number(s): 21.60.Jz, 21.10.Jx

\section{INTRODUCTION}

Recent interest in the spectroscopic factors for the removal of low-lying $p_{1 / 2}$ and $p_{3 / 2}$ strength from ${ }^{16} \mathrm{O}$ points to a substantial discrepancy between experiment [1] and theory [2-13]. The contribution to the reduction of these spectroscopic factors due to short-range correlations is important and is uniformly calculated to be about $10 \%$ in ${ }^{16} \mathrm{O}$ using different realistic interactions and theoretical approaches. The best agreement with the data is obtained when also longrange correlations are included in the calculations $[5,6,9,10]$. The results of Ref. [9] demonstrate a particularly strong correlation between the theoretically calculated excitation spectrum of ${ }^{16} \mathrm{O}$ and the resulting one-hole spectral function, which describes the excitations of the residual $A-1$ nucleus. The appearance of an additional $p_{3 / 2}$ fragment at small missing energy when a low-lying excited $0^{+}$state is obtained is one such correlation [9]. Another one is the appearance of low-lying $s_{1 / 2}$ and $d_{5 / 2}$ strength when the first $3^{-}$state in ${ }^{16} \mathrm{O}$ is correctly described $[6,9]$. Both features are in accordance with the experimental data [1].

The calculation of Ref. [9] employed a random phase approximation (RPA) approach to describe the excitation spectrum of ${ }^{16} \mathrm{O}$. The quality of the spectrum in this approach is, however, quite inadequate. Even with realistic $G$ matrices as residual particle-hole $(p-h)$ interaction, one generates at most a few low-lying collective isoscalar states of negative parity $\left(3^{-}\right.$and $\left.1^{-}\right)$, and no low-lying isoscalar positiveparity states are obtained [14]. These considerations suggest that an adequate description of the single-particle ( $\mathrm{sp}$ ) frag-

\footnotetext{
*Email address: barbieri@triumf.ca; http://www.triumf.ca/people/ barbieri

†Email address: wimd@wuphys.wustl.edu; http:// www.physics. wustl.edu/ wimd
}

mentation, at low energy, requires a better description of the excited states within the same framework of self-consistent Green's function (SCGF).

This improvement of the SCGF method is also important because it can be applied to heavier nuclei. This is possible since the method describes the features of the low-energy spectrum in terms of interactions between a relatively small number of quasiparticles and collective excitations. These modes represent the experimentally observed excitations of the system, and therefore they include both the effects of short- and long-range correlations. The fragmentation of the sp strength is thus self-consistently included. Early applications to ${ }^{48} \mathrm{Ca}$ and ${ }^{90} \mathrm{Zr}$ have been reported in Refs. [15,16]. These calculations did not include self-consistency, but nevertheless, were able to yield a reasonable description of giant resonances and Gamow-Teller states as well as the low-lying collective states. Moreover, a formalism based on the BetheSalpeter equation for $p$ - $h$ excitations can be extended to include the effects of the continuum [17]. Although the SCGF method has only been applied in doubly closed-shell nuclei, it is feasible that increased computational power will allow applications to open-shell systems. An exploratory calculation for semimagic isotopes of $\mathrm{Ni}$ and $\mathrm{Sn}$ was reported in Ref. [18]. In the near future a huge amount of data will become available for unstable nuclei from radioactive beam facilities. For this reason it is important to develop techniques that are flexible enough to describe all the above effects.

In this paper, we report on a first step toward an extension of the SCGF formalism in this direction. We chose to use ${ }^{16} \mathrm{O}$ as a test system for the following reasons. First, a successful calculation for this nucleus will help in resolving the aforementioned discrepancies for the spectroscopic factors. Second, this system has been studied by various approaches in the past. From the results of these calculations one can infer the relevant physical ingredients to be included in the SCGF method. Third, since the spectrum of ${ }^{16} \mathrm{O}$ is rather 

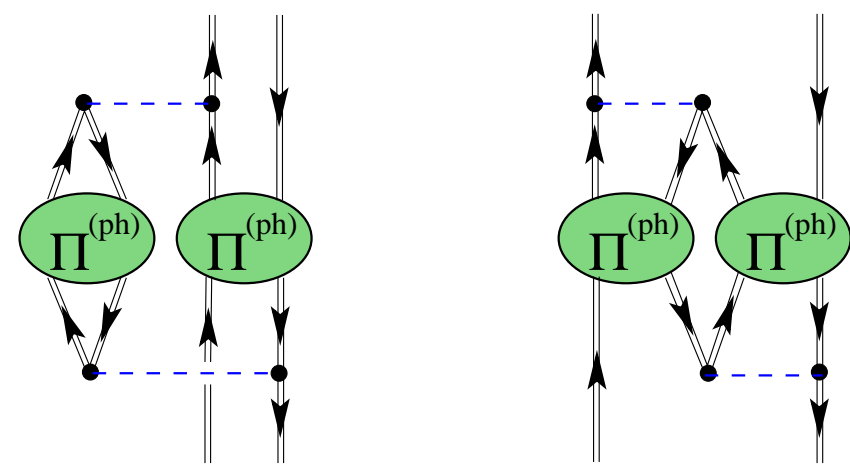

FIG. 1. Examples of contributions involving the coupling of two independent $p$ - $h$ phonons. In total, there are 16 possible diagrams of this type, obtained by considering all the possible couplings to a $p$ - $h$ state. The two-phonon ERPA equations (9) sum all of these contributions in terms of dressed sp propagators.

complicated, meeting the challenge of describing this system will virtually guarantee good results when the same method is applied to heavier nuclei.

Shell-model calculations for this nucleus have been reported in Refs. [19-22], which indicate the particular importance of four-particle-four-hole $(4 p-4 h)$ admixtures to the $0 p-0 h$ ground state in generating the first excited $0^{+}$state. Other positive-parity states at low energy are dominated by $2 p-2 h$ components, as indirectly confirmed by inelastic electron scattering [23]. Some of this resulting physics was anticipated in terms of deformation effects that simulate these types of many-particle many-hole admixtures [24]. A complementary point of view is given by the interacting boson model of Refs. [25,26]. There, the low-lying positive parity states are understood in terms of the coupling of $3^{-}$ and $1^{-}$isoscalar states. One should note that, due to the predominant $1 p-1 h$ nature of these excitations, the coupling of different phonons generates the $n p-n h$ configurations relevant to this problem. In Refs. [25,26], the $3^{-}$and $1^{-}$states were used as phenomenological boson degrees of freedom to generate the spectrum of the nucleus. The connection with the underlying fermionic description was indicated but not explored completely. This relation was considered in Ref. [27]. There it was argued that the microscopic $p$ - $h$ interaction contains two-phonon exchange contributions that include the actually observed low-lying states of ${ }^{16} \mathrm{O}$ themselves, thereby generating the correct number of low-lying states observed at low excitation energy.

In this work we begin to implement the physical ingredients proposed in Refs. $[25,26]$ by extending the RPA to include the coupling to two-phonon states. The basic idea of the present approach is illustrated in Fig. 1. This figure depicts the coupling of a $p-h$ state to two intermediate phonons that are described by the $p-h$ propagator itself. If the two intermediate phonons have been computed using the RPA equations, they will already provide a reasonable description of the low-lying collective isoscalar $3^{-}$and $1^{-}$excited states. These phonons can be sufficient to generate the quantum numbers of the most important positive-parity states. Particularly relevant is the coupling of two $3^{-}$phonons, $3^{-} \otimes 3^{-}=0^{+} \oplus 2^{+} \oplus 4^{+} \oplus 6^{+}$, which represent some of the correlated $2 p-2 h$ states of ${ }^{16} \mathrm{O}$. In the framework of SCGF, one employs dressed sp propagator in the construction of the microscopic phonons. This results in a dressed RPA (DRPA) approach for the $p-h$ calculation and in its extension to the coupling to two-phonon states. In the present paper, we employ the self-consistent sp propagator of ${ }^{16} \mathrm{O}$ computed in Refs. $[28,9]$. It should be noted that the incorporation of all the $4 p-4 h$ effects in the present formalism requires a full four-phonon calculation. For this reason, a complete resolution in terms of a microscopic description of the spectrum may only be partially successful.

We note that there has been tremendous progress in recent years in the microscopic description of $p$-shell nuclei using the Green's function Monte Carlo and no-core shell-model methods [29-31]. A possible application of the no-core shell model to ${ }^{16} \mathrm{O}$ would properly include such $4 p-4 h$ effects. However, the description of spectroscopic factors would still require the construction of effective operators to include the effects of short-range correlations on these quantities whereas these are automatically included in the SCGF method.

The paper is organized as follows. Section II introduces the formalism to account for two-phonon coupling in the calculation of the $p-h$ propagator. The approach employed here is based on a formalism first introduced by Baym and Kadanoff for the description of response functions in a many-body system at finite temperature [32-34]. This framework provides a procedure to construct the effective interaction of the $p$ - $h$ Bethe-Salpeter equation that generalizes the (D)RPA approach. This method is described in Sec. II A and the resulting equations in Sec. II B. More technical details are left to the Appendix. Section III describes the results for the spectrum within the current approximation scheme. Section IV is devoted to a study of convergence properties related to the number of two-phonon configurations included, and the role of time-inversion diagrams. In Sec. IV A we discuss the possible appearance of instabilities, in particular for the $0^{+}$state, which was also observed in Ref. [9]. Conclusions are drawn in Sec. V.

\section{EXTENSION OF THE $p$ - $h$ (D)RPA FORMALISM}

In this work the central quantity of interest is the two-time polarization propagator, whose Lehmann [35] representation is given by

$$
\Pi_{\alpha \beta, \gamma \delta}(\omega)=\sum_{n \neq 0} \frac{\left(\mathcal{Z}_{\alpha \beta}^{n}\right)^{*} \mathcal{Z}_{\gamma \delta}^{n}}{\omega-\varepsilon_{n}^{\pi}+i \eta}-\sum_{n \neq 0} \frac{\mathcal{Z}_{\beta \alpha}^{n}\left(\mathcal{Z}_{\delta \gamma}^{n}\right)^{*}}{\omega+\varepsilon_{n}^{\pi}-i \eta} .
$$

In Eq. (1), the poles and residues contain the information on the response and excitation energies of the system with $A$ particles in terms of the quantities

$$
\begin{gathered}
\mathcal{Z}_{\alpha \beta}^{n}=\left\langle\Psi_{n}^{A}\left|c_{\alpha}^{\dagger} c_{\beta}\right| \Psi_{0}^{A}\right\rangle, \\
\varepsilon_{n}^{\pi}=E_{n}^{A}-E_{0}^{A},
\end{gathered}
$$

where $E_{n}^{A}$ and $\left|\Psi_{n}^{A}\right\rangle$ are the exact energies and eigenstates of the $A$-particle system, the subscript 0 refers to the ground 


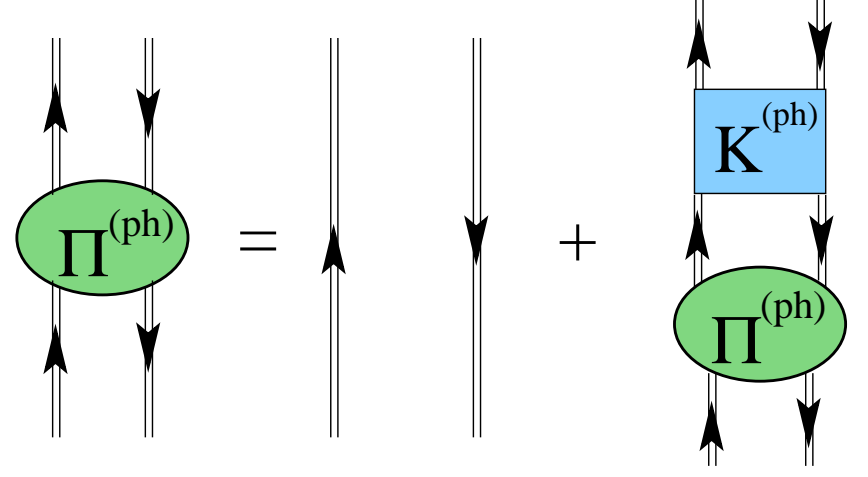

FIG. 2. Bethe-Salpeter equation for the $p-h$ polarization propagator. No specific time direction has to be assumed for these diagrams.

state, and $c_{\alpha}^{\dagger}\left(c_{\alpha}\right)$ is the creation (annihilation) operator of a particle in the state $\alpha$. For a clearer discussion of the formalism employed in this work, it is useful to first give a description of the relevant contributions in terms of Feynman diagrams.

\section{A. Diagrammatic contributions}

The exact $p$ - $h$ propagator (1) is a solution of the BetheSalpeter equation, depicted in Fig. 2 [36,37]. This equation can be written schematically as

$$
\Pi=\Pi^{f}+\Pi^{f} K^{(p-h)} \Pi
$$

where $\Pi^{f}$ represents the free propagation of a quasiparticle and a quasihole in the nuclear medium and the $p$ - $h$ kernel $K^{(p-h)}$ is, in general, a four-time quantity. According to the Baym-Kadanoff procedure, a solution for $\Pi$ is obtained by first generating a self-consistent solution of the sp propagator using a given choice of the self-energy. From the functional derivative of this (self-consistent) self-energy with respect to the corresponding sp propagator, one then obtains the irreducible $p-h$ interaction $K^{(p-h)}$ that generates the corresponding conserving approximation for the $p-h$ propagator (when used in the Bethe-Salpeter equation). The standard RPA approach is derived by applying this procedure to the HartreeFock (HF) sp propagator and self-energy. This corresponds to approximating $K^{(p-h)}$ with the bare interaction $V$ and employing bare (HF) sp propagators as external lines. Equation (3) then generates the RPA series of ring diagrams shown in Fig. 3.

The extension of the RPA formalism, proposed and implemented by Brand et al. $[15,16]$, was suggested by the observed fragmentation of the sp strength and the necessity to go beyond a lowest-order self-energy for a commensurate theoretical description. There, the Baym-Kadanoff procedure is applied to a second-order approximation for the selfenergy. This yields contributions to the kernel $K^{(p-h)}$, which automatically include all the terms which couple the $p-h$ states to the $2 p-2 h$ ones, in accordance with the Pauli principle for the latter states. Thus, this formalism takes into account the mixing with $2 p-2 h$ configurations in the construction of the $p-h$ propagator [the diagram of Fig. 4(a)

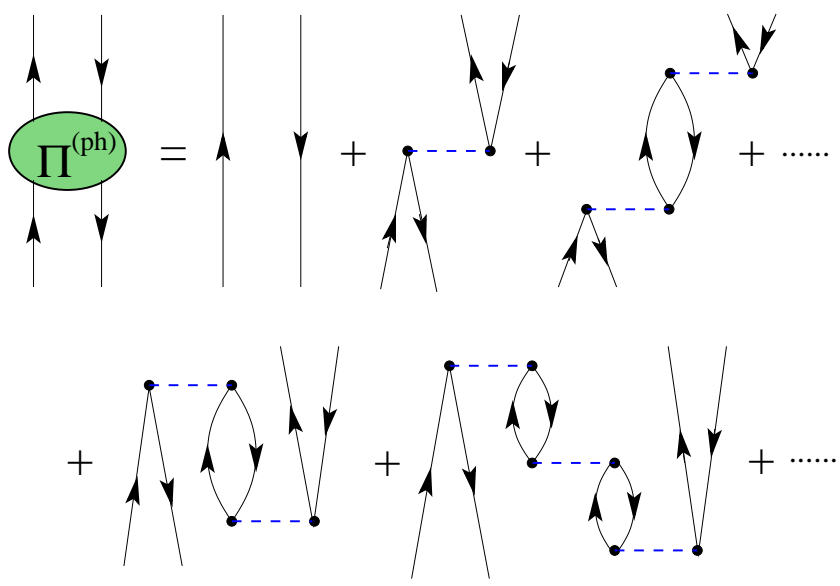

FIG. 3. Diagrammatic expansion of the standard RPA equation. An explicit time direction is assumed for the diagrams of this figure.

gives an example]. In the work of Brand et al. only a singlepole approximation to the self-consistent propagators was employed. It is the aim of the present work to take the effects of the sp fragmentation more completely into account, and therefore a fully dressed sp propagator must be used. The one employed in the present work was obtained in Ref. [9] by means of a Faddeev expansion for the nuclear self-energy. When one applies the Baym-Kadanoff prescription to the latter self-energy, a large set of contributions to $K^{(p-h)}$ is generated [38]. According to Ref. [27], one may expect that the most important of these terms involve the couplings to two $p$ - $h$ phonons as depicted in Fig. 1. It is not difficult to see that different diagrams, similar to those in Fig. 1, can be obtained through Pauli exchange of the phonon's external lines. In total, there are 16 such possible contributions, corresponding to all the possibilities of connecting two $p-h$ phonons to a $p$ - $h$ state by means of a single interaction, both in the upper and lower part of the diagram. It is this approximation to the irreducible interaction that will be pursued in the present work.

An additional ingredient entering the $2 p-2 h$ extended RPA (ERPA) of Refs. [15,16,39] requires further discussion. This involves diagrams similar to the one in Fig. 4(b), which
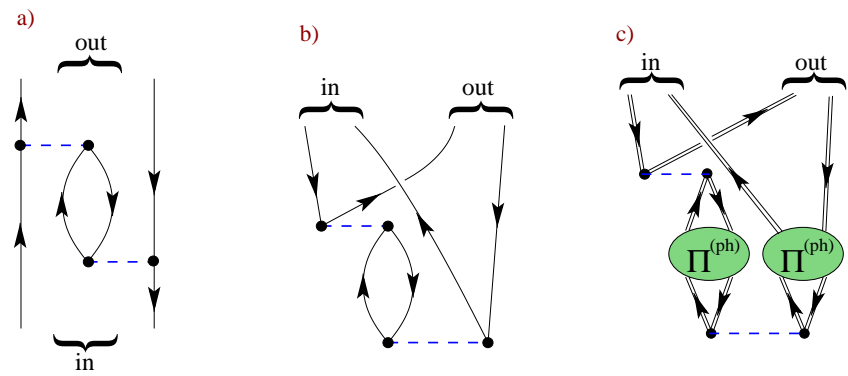

FIG. 4. Example of direct (a) and time inversion (b) diagrams that appear in the standard ERPA expansion. Both diagrams (a) and (b) come from the same four-time screening diagram. The last picture in part (c) shows the corresponding two-phonon extension of the time-inversion contribution. Note that the diagram (b) generates Pauli exchange corrections to the last diagram shown in Fig. 3. 
are obtained from the $2 p-2 h$ contribution by inverting the sense of propagation of either the incoming or the outgoing $p-h$ pair. These diagrams involve higher excitations (at least $3 p-3 h$, when combined in the expansion of Fig. 3) and are expected to give rather small contributions. Nevertheless, they represent corrections to the terms of the $p$ - $h$ interactions, which control the RPA correlations, and they also add Pauli corrections to the RPA expansion of Fig. 3 at the $3 p-3 h$ level. In Ref. [16] it was found that they play a role in stabilizing some particular solutions.

The present calculation includes both the direct twophonon contributions of the type depicted in Fig. 1 and the diagrams similar to the one in Fig. 4(c). Obviously, all the $2 p-2 h$ ERPA contributions are incorporated in this approach. Moreover, full two-phonon configurations are accouned for, and a SCGF approach is applied. Thus, the present formalism is an extension of (and goes well beyond) the calculations of Refs. $[15,16,39,40]$. However, to avoid complications in the notation, we will still refer to it as "extended RPA," or as "two-phonon ERPA" whenever confusion may arise.

The Baym-Kadanoff procedure also generates other twophonon contributions, for example, those coupling a $p-p$ and a $h-h$ phonon. These could be mixed with the presently considered configurations by means of an all-order expansion of the Faddeev-Yakubovsky type. Given our present knowledge, such a massive resummation of diagrams does not appear to be relevant for the understanding of the spectrum of ${ }^{16} \mathrm{O}$. Such a study is, in any case, beyond the scope of the present paper.

\section{B. Two-phonon contributions to the $p-h$ propagator}

The usual DRPA equations are obtained from Eq. (3) by choosing $K_{\alpha \beta, \gamma \delta}^{(p-h)}=V_{\alpha \delta, \beta \gamma}$, as mentioned above, and by keeping dressed propagators as external lines. In this way, one is left with only two-time quantities and, after Fourier transformation, the DRPA equation becomes

$$
\begin{aligned}
\Pi(\omega)_{\alpha \beta, \gamma \delta}= & \Pi_{\alpha \beta, \gamma \delta}^{f}(\omega) \\
& +\sum_{\mu, \rho, \nu, \sigma} \Pi_{\alpha \beta, \mu \rho}^{f}(\omega) V_{\mu \sigma, \rho \nu} \Pi_{\nu \sigma, \gamma \delta}(\omega),
\end{aligned}
$$

where all the indices and summations are shown explicitly. In Eq. (4), the free polarization propagator $\Pi^{f}(\omega)$ is also a two-time quantity, with the following Lehmann representation:

$$
\begin{aligned}
\Pi_{\alpha \beta, \gamma \delta}^{f}(\omega)= & \sum_{n, k} \frac{\left(\mathcal{X}_{\alpha}^{n} \mathcal{Y}_{\beta}^{k}\right) * \mathcal{X}_{\gamma}^{n} \mathcal{Y}_{\delta}^{k}}{\omega-\left(\varepsilon_{n}^{+}-\varepsilon_{k}^{-}\right)+i \eta} \\
& +\sum_{k, n} \frac{\mathcal{Y}_{\alpha}^{k} \mathcal{X}_{\beta}^{n}\left(\mathcal{Y}_{\gamma}^{k} \mathcal{X}_{\delta}^{n}\right)^{*}}{\omega+\left(\varepsilon_{n}^{+}-\varepsilon_{k}^{-}\right)-i \eta}
\end{aligned}
$$

where $\mathcal{X}_{\alpha}^{n}=\left\langle\Psi_{n}^{A+1}\left|c_{\alpha}^{\dagger}\right| \Psi_{0}^{A}\right\rangle\left(\mathcal{Y}_{\alpha}^{k}=\left\langle\Psi_{k}^{A-1}\left|c_{\alpha}\right| \Psi_{0}^{A}\right\rangle\right)$ are the spectroscopic amplitudes for the excited states of a system with $A+1(A-1)$ particles and the associated poles $\varepsilon_{n}^{+}$
$=E_{n}^{A+1}-E_{0}^{A}\left(\varepsilon_{k}^{-}=E_{0}^{A}-E_{k}^{A-1}\right)$ correspond to the excitation energies with respect to the $A$-body ground state. The indices $n$ and $k$ label the eigenstates of the systems with $A \pm 1$ particles and enumerate the fragments associated with the one-particle and one-hole excitations, respectively. When a dressed propagator is used as input, its one-body overlap functions and quasiparticle energies already contain information about the coupling of sp motion to $2 p-1 h, 2 h-1 p$, and more complex configurations. As a consequence, contributions beyond the $1 p-1 h$ case are already included in $\Pi^{f}(\omega)$.

Methods based on an RPA-like expansion produce an infinite series of diagrams in which the direction of propagation can be reversed from backward to forward and vice versa. In the case of standard (D)RPA, the interaction kernel is simply given the potential $V$ and it is the same for every contribution to the diagrammatic expansion. Therefore the usual (D)RPA equation can be written in compact form, as in Eq. (4). This is no longer true when one aims to include additional contributions, and at the same time insists on working with two-time quantities. The two-phonon diagrams of Figs. 1 and 4(c) have different analytical expressions. As a consequence, one first needs to separate all the four possible time directions of the kernel $K^{(p-h)}$-forward to forward, backward to backward, and the two time-inversion casesbefore including the relevant diagrams in the Bethe-Salpeter equation (3). This can be achieved by splitting the free $p-h$ propagator (5) into its forward- and backward-going parts, denoted by $>$ and $<$, respectively,

$$
\Pi^{f}(\omega) \rightarrow \Pi^{f>}(\omega)+\Pi^{f<}(\omega)
$$

By performing this substitution in Eq. (3), one obtains a similar separation for the complete $p$ - $h$ propagator,

$$
\Pi(\omega) \rightarrow \Pi^{>}(\omega)+\Pi^{<}(\omega),
$$

where $>$ and $<$ now refer to the sense of propagation of the final lines only. Suppressing the indices and summations, one obtains

$$
\begin{aligned}
& \Pi^{>}(\omega)=\Pi^{f>}(\omega)+\Pi^{f>}(\omega) K^{(p-h)} \Pi(\omega), \\
& \Pi^{<}(\omega)=\Pi^{f<}(\omega)+\Pi^{f<}(\omega) K^{(p-h)} \Pi(\omega) .
\end{aligned}
$$

The last step consists in substituting Eq. (7) into Eq. (8) and approximating each component of the $p$ - $h$ kernel $K^{(p-h)}$ with the sum of the bare interaction and the corresponding twophonon contributions. The result corresponds to the ERPA equations given by

$$
\begin{aligned}
\Pi^{>}(\omega)= & \Pi^{f>}(\omega)+\Pi^{f>}(\omega)\left\{\left[V+W^{>}(\omega)\right] \Pi^{>}(\omega)\right. \\
& \left.+\left(V+H^{>},<\right) \Pi^{<}(\omega)\right\}, \\
\Pi^{<}(\omega)= & \Pi^{f<}(\omega)+\Pi^{f<}(\omega)\left\{\left(V+H^{<,>}\right) \Pi^{>}(\omega)\right. \\
& \left.+\left[V+W^{<}(\omega)\right] \Pi^{<}(\omega)\right\} .
\end{aligned}
$$

In Eq. (9), $W^{>}(\omega)$ represents the contribution of all the 16 two-phonon diagrams, Fig. 1, in the forward direction. $W^{<}(\omega)$ corresponds to the contributions connecting the 
backward-going terms. Analogously, $H^{>,<}$represent the sum of the diagrams in which a backward-going $h-p$ state is inverted in a forward-going $p$ - $h$ one [Fig. 4(c) illustrates such a case], while $H^{>},<$is the time-reversed contribution.

The practical implementation of Eqs. (9) requires additional manipulation to treat the freely propagating lines in Figs. 1 and 4(c). This situation is completely analogous to the one already discussed in Refs. [28,41] for the $2 p-1 h$ expansion of the self-energy. Specific details for the ERPA equation (9) are given in the sections in the Appendix. The following section reports on the application of this formalism to ${ }^{16} \mathrm{O}$.

\section{RESULTS}

As in the work of Ref. [9], the ERPA equations were solved within a model space, consisting of a finite set of harmonic oscillator states. All the first four major shells (from $1 s$ to $2 p 1 f$ ) plus the $1 g_{9 / 2}$ where included to account for the sp orbitals that are most relevant for low-lying excitations. The harmonic oscillator parameter was chosen to be $b=1.76 \mathrm{fm}$. As a consequence of the truncation of the model space a Brueckner $G$ matrix was used as a microscopic effective interaction. This $G$ matrix was derived from a Bonn$C$ potential [42] and computed according to Ref. [43].

The contributions of two-phonon states were first studied by solving the ERPA equation (9) with an independentparticle model (IPM) propagator. This propagator was constructed from a Slater determinant composed of the lowest occupied harmonic oscillator (h.o.) orbitals in the model space. Where the effects of nuclear fragmentation were included, the calculations employed the fully dressed sp propagator computed in Ref. [9]. This propagator contains no more than two main quasihole (quasiparticle) fragments for each state in the $p(s d)$ shell. For these orbits, the sp propagator can therefore be well represented by means of one or two principal fragments and an effective pole that accounts for strength far from the Fermi energy. For quasiparticle states associated with the $p f$ shell, instead, the fragmentation is more substantial [38] and the self-consistent propagator was approximated by including the most important poles (up to four main fragments for the $p_{3 / 2}$ ) plus two effective ones that gather all the background strength. We have checked that the results are not sensitive to the details of the fragmentation of the $1 f$ orbitals. For the $2 p$ orbitals, we found that the strength distribution is sufficiently represented by the adopted prescription, and further discuss the sensitivity to the results in Sec. IV A.

In both cases (IMP and dressed inputs), the standard (D)RPA equation was solved first, in order to generate the $p$ - $h$ phonons that entered the final two-phonon ERPA calculation. When coupling different phonons, only the lowest few states of ${ }^{16} \mathrm{O}$ have the right quantum numbers, and energies low enough, to generate two-phonon contributions with unperturbed energy below $20 \mathrm{MeV}$. These are the only states relevant for our purposes [27]. In practice, the calculations were performed by including, in each channel, all twophonon configurations up to $30 \mathrm{MeV}$. The stability tests reported in Sec. IV C demonstrate that, for the case of a

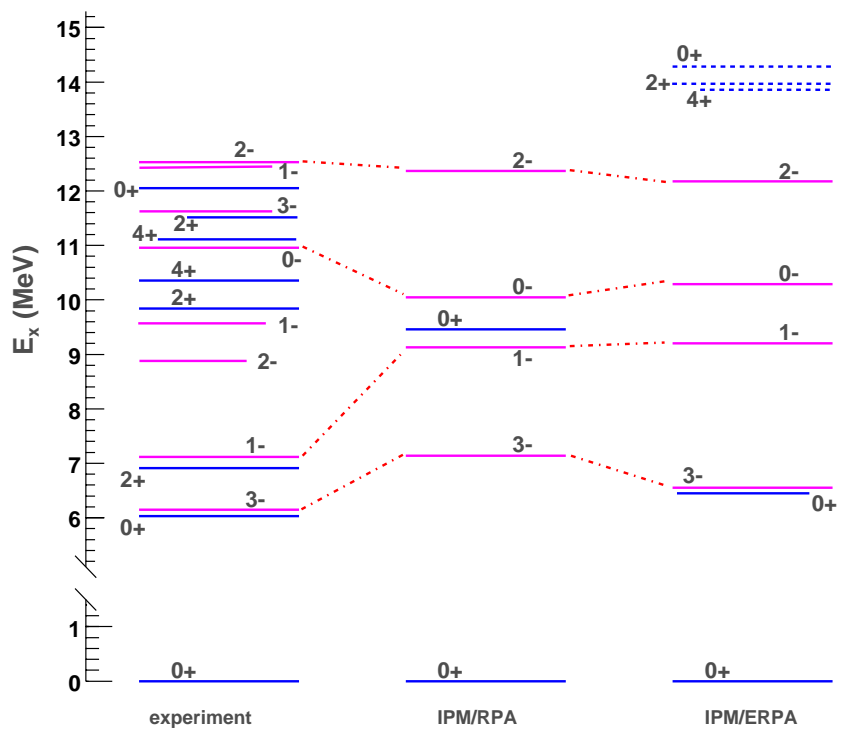

FIG. 5. Results for the two-phonon ERPA propagator of ${ }^{16} \mathrm{O}$ with an h.o. IPM input propagator, last column. The spectrum in the middle is obtained by solving the standard RPA problem and is employed, as is, to generate two-phonon contributions to the ERPA equation. The excited states indicated by dashed lines are those for which the ERPA equation predicts a total spectral strength $Z_{n_{\pi}}$ lower than $10 \%$. The first column reports the experimental results taken from Ref. [44].

dressed input propagator, higher two-phonon contributions do not change the results for the low-lying states appreciably.

\section{A. Results for the particle-hole propagator}

The results obtained from the h.o. input propagator are displayed in Fig. 5. The isoscalar eigenvalues obtained for energies below $15 \mathrm{MeV}$ are displayed, for both the standard RPA and for the ERPA calculation. The ERPA calculation produces lower energies for some of the states that were already obtained in RPA. Both $3^{-}$and $0^{+}$shifted down to about $0.5 \mathrm{MeV}$ above the experimental energy. As discussed further in Sec. IV C, this is to be considered rather fortuitous and one must remember that the dressing of the sp propagator, which has the effect to raise these eigenvalues, has not been taken into account yet. Still, this result indicates that correlations between $p-h$ and two-phonon states can be substantial and go in the right direction in explaining the experimental results.

It is also worth considering the total $p-h$ spectral strength of these states obtained by summing the corresponding amplitudes, Eq. (2), as follows:

$$
Z_{n_{\pi}}=\sum_{\alpha \beta}\left|\mathcal{Z}_{\alpha \beta}^{n}\right|^{2}
$$

Results for both the excitation energy and $Z_{n_{\pi}}$ of the principal levels in Fig. 5 are given in Table I. For the ERPA case, also the fraction of quasiparticle-quasihole and two-phonon configuration, which appear in the wave function are shown. Note that $Z_{n_{\pi}}$ is substantially bigger than 1 for the $3^{-}$ 
TABLE I. Excitation energy and total spectral strengths obtained for the principal solutions of the RPA and ERPA equations. For the ERPA case the total fraction of $p-h$ and two-phonon contributions are also shown. An IPM input sp propagator was used to generate these results.

\begin{tabular}{ccccccc}
\hline \hline \multirow{2}{*}{\begin{tabular}{c}
\multirow{2}{*}{0} \\
$J^{\pi}$
\end{tabular}} & \multicolumn{2}{c}{ h.o./RPA } & \multicolumn{4}{c}{ h.o./ERPA } \\
& $\varepsilon_{n_{\pi}}$ & $Z_{n_{\pi}}$ & $\varepsilon_{n_{\pi}}$ & $Z_{n_{\pi}}$ & $p-h(\%)$ & $2 \Pi(\%)$ \\
\hline $2^{+}$ & 22.86 & \multirow{2}{*}{1.039} & 23.13 & 0.823 & 79 & 21 \\
$2^{+}$ & & & 21.30 & 0.133 & 13 & 87 \\
$2^{+}$ & & & 19.11 & 0.118 & 11 & 89 \\
$0^{+}$ & & & 14.28 & 0.010 & 1 & 99 \\
$2^{+}$ & & & 13.91 & 0.041 & 4 & 96 \\
$4^{+}$ & & & 13.87 & 0.028 & 3 & 97 \\
$1^{-}$ & 9.13 & 1.027 & 9.20 & 1.075 & 99.7 & 0.3 \\
$3^{-}$ & 7.14 & 1.258 & 6.55 & 1.269 & 97 & 3 \\
$0^{+}$ & 9.46 & 1.582 & 6.52 & 1.820 & 80 & 20 \\
\hline \hline
\end{tabular}

and $0^{+}$states, and that these values increase further for the ERPA results. This signals an increase of the collective character of these solutions, which may lead to an instability of the RPA equations for interactions that are even more attractive. The two-phonon ERPA approach generates a triplet of states at about $14 \mathrm{MeV}$, with quantum numbers $0^{+}, 2^{+}$, and $4^{+}$. However, these levels are almost exclusively composed of two-phonon contributions and contain only small admixtures of $p$ - $h$ states, resulting in a small $p$ - $h$ spectral strength $Z_{n_{\pi}}$. The quantum numbers and energies of these states indeed correspond closely to those obtained by coupling two $3^{-}$RPA phonons, each at $7.14 \mathrm{MeV}$. We note that a similar triplet is found experimentally at 12.05, 11.52, and 11.10 $\mathrm{MeV}$, which also corresponds to twice the experimental energy of the first $3^{-}$phonon. The first experimental $2^{+}$is found at lower energy, and its spectral strength is known to have relevant $p$ - $h$ components [23]. Thus it cannot be identified with any of the above two-phonon contribution. For all the lowest states that are not already reproduced by standard RPA a very small $p$ - $h$ spectral strength has been found, due to a general lack of mixing between the $p$ - $h$ and the twophonon configurations. Of interest is also the $2^{+}$that represents the giant quadrupole resonance at $20.7 \mathrm{MeV}$. In this case RPA and ERPA give 22.9 and $23.3 \mathrm{MeV}$ for the main peak but with a lower $Z_{n_{\pi}}$ in the second case. For this state, part of the $p$ - $h$ strength (about 20\%) is shifted to two-phonon configurations representing the expected fragmentation of the giant resonance.

Figure 6 and Table II show the analogous results when dressed sp propagators are used as input. In general, the main effect of fragmentation is to screen the nuclear interaction as a consequence of the quenching of spectroscopic factors for the input sp propagator. For the DRPA case, this results in increasing the lowest $3^{-}$and $1^{-}$solutions by $\sim 2 \mathrm{MeV}$. More substantial is the effect on the lowest $0^{+}$state with a predominantly $p$ - $h$ character which rises to about $17 \mathrm{MeV}$, confirming the sensitivity of this state to details of the fragmentation and the strength of the nuclear interaction. Unlike the IPM case, we have chosen to solve the two-phonon

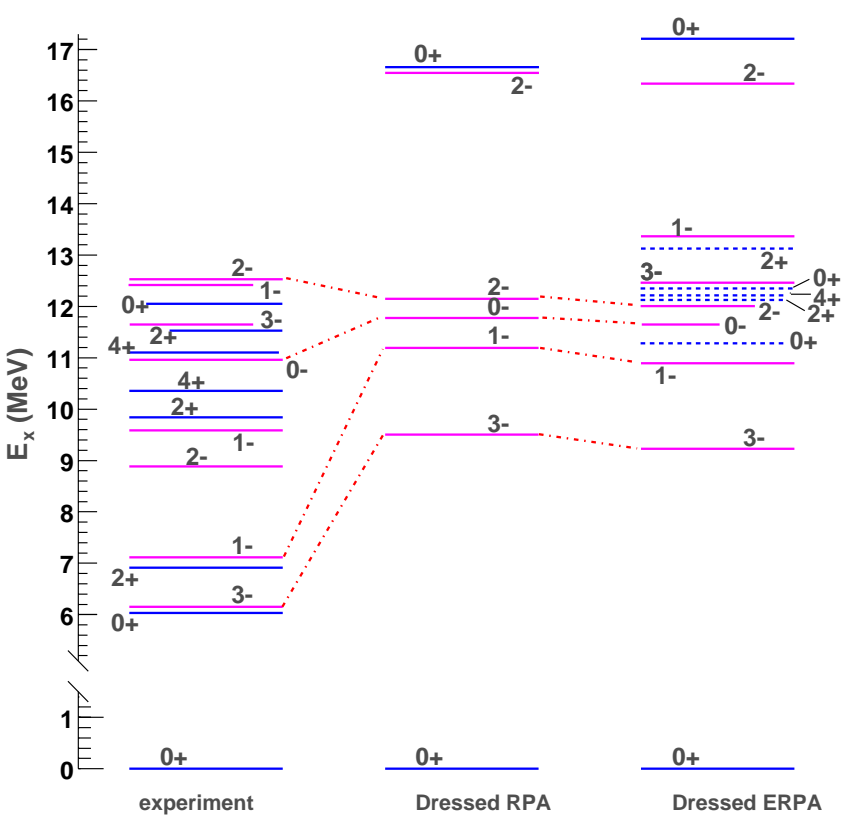

FIG. 6. Results for the DRPA and the two-phonon ERPA propagator of ${ }^{16} \mathrm{O}$ with a dressed input propagator from Ref. [9], middle, and last column, respectively. In solving the ERPA equation, the lowest $3^{-}, 1^{-}$, and $0^{+}$levels of the DRPA propagator were shifted to their experimental energies. All other DRPA solutions were left unchanged. The excited states indicated by dashed lines are those for which the (E)RPA equation predicts a total spectral strength $Z_{n_{\pi}}$ lower than $10 \%$. The first column reports the experimental results [44].

ERPA by first shifting the lowest solution for both the $0^{+}$, $3^{-}$, and $1^{-}$states down to their relative experimental energies. This has the advantage of lowering the most important two-phonon configurations and allows us to investigate their interplay with the $p$ - $h$ ones.

As can be seen in Fig. 6, the ERPA equations still generate a triplet of $0^{+}, 2^{+}$, and $4^{+}$states at twice the energy of the first $3^{-}$phonon. Due to the screening of the $p-h$ interaction, these states mix very little with the $p$ - $h$ configurations yielding an almost degenerate triplet. Table II also gives a comparison between the total two-phonon content of the states and the individual contributions of the most important configurations. This decomposition demonstrates that this triplet is formed by pure $3^{-} \otimes 3^{-}$states. This observation is in accordance with the $2 p-2 h$ character of these states $[23,45]$. Since the overall energy of these states approximately agrees with experiment, one can expect that this calculation correctly represents the bulk properties of their wave functions. Nevertheless, it is clear that an additional interaction is needed in order to split this triplet as observed experimentally. It should be noted that no solution that can be identified with the $2^{+}$and $4^{+}$levels at 9.8 and $10.4 \mathrm{MeV}$. The $2^{+}$level has been interpreted in terms of an $\alpha$ particle rotating around an excited ${ }^{12} \mathrm{C}$ core [23], and therefore involves correlations that may go well beyond the present calculation. With regard to the $2^{+}$strength around $20 \mathrm{MeV}$, we note that the sp fragmentation included in the DRPA equation already generates a distribution of $2^{+}$strength. Table II con- 
TABLE II. Excitation energy and total spectral strengths obtained for the principal solutions of DRPA and two-phonon ERPA equations. A dressed sp propagator was employed. The total contribution of $p-h$ and two-phonon states of the ERPA solutions are shown. For states below $15 \mathrm{MeV}$, the columns on the right side give the individual contributions of all the relevant two-phonon contributions. The sum of these terms for the states that are listed does not exceed $1 \%$.

\begin{tabular}{|c|c|c|c|c|c|c|c|c|c|c|}
\hline \multirow{2}{*}{$\begin{array}{c}T=0 \\
J^{\pi}\end{array}$} & \multicolumn{2}{|c|}{ Dressed/DRPA } & \multicolumn{4}{|c|}{ Dressed/ERPA } & \multirow{2}{*}{$\begin{array}{c}\left(0^{+}\right)^{2} \\
(\%)\end{array}$} & \multirow{2}{*}{$\begin{array}{c}\left(3^{-}\right)^{2} \\
(\%)\end{array}$} & \multirow{2}{*}{$\begin{array}{c}\left(0^{+}, 3^{-}\right) \\
(\%)\end{array}$} & \multirow{2}{*}{$\begin{array}{c}\left(0^{+}, 1^{-}\right) \\
(\%)\end{array}$} \\
\hline & $\varepsilon_{n}^{\pi}$ & $Z_{n_{\pi}}$ & $\varepsilon_{n}^{\pi}$ & $Z_{n_{\pi}}$ & $p-h(\%)$ & $2 \Pi(\%)$ & & & & \\
\hline $2^{+}$ & 23.77 & 0.468 & 23.52 & 0.123 & 26 & 74 & & & & \\
\hline $2^{+}$ & & & 22.96 & 0.341 & 78 & 22 & & & & \\
\hline $2^{+}$ & 20.59 & 0.269 & 20.42 & 0.255 & 98 & 2 & & & & \\
\hline $1^{-}$ & & & 13.37 & 0.148 & 21 & 79 & & & & 79 \\
\hline $3^{-}$ & & & 12.35 & 0.113 & 16 & 84 & & & 84 & \\
\hline $0^{+}$ & & & 12.15 & 0.001 & 1 & 99 & 3 & 96 & & \\
\hline $4^{+}$ & & & 12.14 & 0.007 & 1 & 99 & & 99 & & \\
\hline $2^{+}$ & & & 12.12 & 0.008 & 1 & 99 & & 98 & & \\
\hline $0^{+}$ & 16.62 & 0.717 & 17.21 & 0.633 & 88 & 12 & 10 & 0.5 & & \\
\hline $0^{+}$ & & & 11.28 & 0.092 & 12 & 88 & 85 & 2 & & \\
\hline $1^{-}$ & 11.19 & 0.720 & 10.90 & 0.680 & 94.1 & 5.9 & & & & 5.8 \\
\hline $3^{-}$ & 9.50 & 0.762 & 9.23 & 0.735 & 95.9 & 4.1 & & & 4.0 & \\
\hline
\end{tabular}

tains a few of these solutions and shows that also for the dressed case a sizable mixing of $p-h$ and two-phonon contributions is obtained at this energy. This mixing generates the spreading of $p$ - $h$ strength over different solutions, consistent with the finite width of such a resonant state. It should be noted, however, that a discrete basis is used here, and therefore it is not possible to properly describe the continuous strength distribution of a giant resonance, for which a continuum or a complex basis should be used [46].

Also interesting are the results for the other isoscalar $0^{+}$ states. The lowest solution with a predominant $p$ - $h$ character, which was found in DRPA at $\sim 17 \mathrm{MeV}$, is obtained at a similar energy but is now characterized by a partial mixing with two-phonon configurations. The $p$ - $h$ and two-phonon contents of this state given in Table II show that this is the result of mixing with the lowest solution, which ends up at $\sim 11 \mathrm{MeV}$. The latter is predominantly a two-phonon state. It is also seen that in both cases the relevant configuration comes from the coupling of two $0^{+}$phonons. Configurations involving two $3^{-}$'s make smaller contributions while the other modes not reported in the table were negligible. We note that the wave functions for these states contain several relevant $p-h$ configurations, obtained from different quasiparticle fragments in the $p f(s d)$ shells combined with quasihole fragments of the $p(s)$ shells. Therefore, the situation is more complicated than the simple picture of only two levels interacting with each other. We observe that the shell-model calculations for the first excited state of ${ }^{16} \mathrm{O}$ give very small contributions from both $0 \hbar \omega$ and $2 \hbar \omega$ configurations and a strong population of $4 \hbar \omega$ states $[21,22]$. On the other hand, inelastic electron scattering experiments [23] clearly excite this state identifying its partial $p-h$ character even though the state is dominated by $4 p-4 h$ components. From the point of view of the SCGF approach, the one-body response is completely described by the (dressed) $p-h$ propagator, Eq. (4), and therefore the total one-body strength must be represented by $Z_{n_{\pi}}$ (10). This observation points to the necessity of a stronger mixing between $p$ - $h$ and two-phonon configurations than obtained in the present calculations. Still, the present results indicate that the two-phonon configuration obtained by coupling the two lowest $0^{+}$phonons plays a role in the structure of the first $0^{+}$state itself. This means that, starting from the dressed $p-h$ admixture contained in it, a fully selfconsistent calculation would also generate some contributions of $2 p-2 h, 4 p-4 h$, and beyond. The calculations of Ref. [25] have shown that the bulk of the $4 p-4 h$ contributions to the first excited state of ${ }^{16} \mathrm{O}$ may come from the coupling of four different phonons with negative parity $\left(3^{-}\right.$and $\left.1^{-}\right)$. However, the self-consistent role of coupling positive-parity states was not conidered in that work. Both these effects, the RPA correlations and the inclusion of the nuclear fragmentation, allow for the-at least partial-inclusion of configurations beyond the $2 p-2 h$ case even when no more than twophonon coupling is considered, as in this paper. A study of the importance of three- and four-phonon configurations within this approach is beyond the scope of the present work.

The principal negative-parity isoscalar states are still shifted down in energy by ERPA over DRPA, as expected, but the improvement is less than $0.5 \mathrm{MeV}$. The low-lying $3^{-}$ and $1^{-}$levels remain substantially above the experimental energy at 9.23 and $10.90 \mathrm{MeV}$, respectively, and more correlations will be needed in order to lower their energy. We note that these wave functions contain a two-phonon admixture obtained by coupling the low-lying $0^{+}$excitation to either the $3^{-}$or $1^{-}$phonons. According to the above interpretation of the first $0^{+}$excited state, this corresponds to the inclusion of $3 p-3 h$ and beyond. At the same time, two additional negative-parity solutions (with two-phonon character) are found at higher energy in accordance with experiment.

The ERPA results for an h.o. input give appropriate corrections to several energy levels. However, such corrections tend to become negligible in the successive calculations employing dressed propagators. Presumably, this happens be- 
cause a sizable part of the correlations, which in the latter case are introduced by ERPA, are already accounted for by the dressing of the sp propagators. In general, the inclusion of fragmentation has also the effect of screening the interaction between $p-h$ and two-phonon configurations. However, the results of Table II show that a considerable mixing between the two can be obtained if all the relevant configurations are sufficiently low in energy, although no substantial mixing between different two-phonon states is seen. According to these considerations, the biggest deficiency of the present approach is probably the lack of an interaction within the multiphonon space. References $[25,26]$ have shown that such correlations are due to $p-p$ and $h-h$ interactions, neglected here, and that their inclusion has important effects on the final result for the spectrum. These terms may considerably lower most of the eigenstates of the ERPA equations. However, a second type of correlation between multiphonon states, which was not accounted for in Refs. [25,26], is the Pauli exchange between the fermion lines included in different phonon propagators. These will probably generate the opposite effect, screening part of the $p-p$ and $h$ - $h$ correlations.

Although the results obtained with a dressed input propagator for the lowest excited states are not completely successful, it must be recognized that the present ERPA calculation does represent a step forward. Indeed, a proper description of two-phonon contributions (at the present level) had not been accomplished before. These states not only account for configurations at the $2 p-2 h$ and higher level, but appear to have some relevance for the description of states at higher energy. In addition, excitations are generated with quantum numbers that cannot be obtained in the standard (D)RPA approach at these low energies. Given the results of the present work, it appears that a calculation including both $p-p$ and $h$ - $h$ correlations, a more complete treatment of Pauli effects and the most relevant configurations up to the fourphonon states can be achieved with the present-day computers. Such a calculation is planned for the future.

\section{STABILITY OF THE RESULTS AND TRUNCATION OF THE MODEL SPACE}

\section{A. RPA instability}

The RPA approach may break down for strongly attractive interactions, by generating an excess of collectivity and lowering the first excited state below the ground-state energy. The inclusion of fragmentation of the sp strength can affect this behavior in several ways. First, both the screening of the nuclear interaction and the splitting of the particle and hole spectral strengths over different fragments tends to stabilize the DRPA equations. Second, correlations lower most of the sp energies in comparison with the IPM. This results in smaller unperturbed $p$ - $h$ energies, and therefore has the opposite effect of pushing the DRPA approach toward instability. Third, the instability can be sensitive to the details of the spectroscopic amplitudes. This last point can be best illustrated by looking at the sp strength with $j^{\pi}=3 / 2^{-}$, to which the present calculation is most sensitive. For the model space employed in this work, a given particle overlap function is a superposition of two h.o. eigenstates,

$$
\psi_{p_{3 / 2}}^{n}(\mathbf{x})=\mathcal{X}_{1 p_{3 / 2}}^{n} \phi_{1 p_{3 / 2}}^{\text {h.o. }}(\mathbf{x}) \pm \mathcal{X}_{2 p_{3 / 2}}^{n} \phi_{2 p_{3 / 2}}^{\text {h.o. }}(\mathbf{x}),
$$

where we have stressed the fact the two components can sum either constructively or destructively. The fragmentation introduces a nonzero $\mathcal{X}_{1 p_{3 / 2}}^{n}$ component even for particle states that in the IPM would be described only by the $2 p_{3 / 2}$ subshell. By considering Eqs. (A14), one can see that the $p-h$ interaction for the DRPA approach can be substantially changed by this new component by virtue of the strong matrix elements of the effective interaction between $1 p_{3 / 2}$ states. In particular, this also applies to the off-diagonal terms that drive the RPA-like correlations. It is also worth noting that the sign of this correction changes accordingly to the relative sign of the components in Eq. (11), therefore pushing the states either towards stability or instability, respectively.

We have observed that choosing a wrong sign for one or more of the $p_{3 / 2}$ particle fragments has drastic effects for the solutions of the isoscalar $0^{+}$channel, with the choice of constructive interference in Eq. (11) leading toward instability of the DRPA approach. For the input propagator employed in this work, all of the background fragments have a similar wave function and a negative or positive interference sign according to whether their sp energy is lower or higher than $20 \mathrm{MeV}$, respectively [38]. Therefore it is natural to collect such background distributions in two different poles, one for each region of missing energy. A similar behavior applies also to the other quasiparticle states in the $2 p$ and $s d$ shells. We have checked that the results of Sec. III are stable with respect to the number of main fragments and effective poles included in each subshell, therefore no instability affects the present work. However, in applications in which the number of poles has to be strongly reduced, it may not be possible to choose effective poles of the sp propagator, which represent the details of the spectral distribution sufficiently accurately. In such a case an instability in the DRPA approach may arise. This situation occurred with a previous DRPA calculation of the isoscalar $0^{+}$channel, reported in Ref. [9], in which a smaller number of fragments was included. Thanks to improvements in the computer code that solves the DRPA problem, it is now possible to consider a sufficiently large number of poles in the sp propagator, and this problem has been overcome.

\section{B. Time-inversion diagrams}

The contribution of two-phonon terms to the timeinversion diagrams of Fig. 4 can, in principle, generate other correction to the interaction between $p-h$ and $h-p$ states, which drives the RPA correlations. Nevertheless, the effect is very small due to the large energy denominators that appear in Eqs. (A18) and (A19). We have tested their influence by neglecting the corresponding terms $H^{>,}<$and $H^{<,>}$in Eq. (9) and report the results in Table III. No appreciable change is generated illustrated by differences with respect to the results of Sec. III of at most $0.1 \%$. It is worth noting that the 
TABLE III. The excitation energy and total spectral strengths discussed in Sec. III are compared to the solutions of the two-phonon ERPA equations by neglecting the time-inversion diagrams of Fig. 4. Both IPM and dressed input propagator cases are displayed. Results obtained within a larger model space $(\rho=2 n+l=5)$ are also shown.

\begin{tabular}{|c|c|c|c|c|c|c|c|c|c|c|c|c|}
\hline \multirow{2}{*}{$\begin{array}{c}T=0 \\
J^{\pi}\end{array}$} & \multicolumn{2}{|c|}{ h.o./ERPA } & \multicolumn{2}{|c|}{$\begin{array}{c}\text { h.o./ERPA } \\
\text { No time inversion }\end{array}$} & \multicolumn{2}{|c|}{$\begin{array}{c}\text { h.o./ERPA } \\
\rho=5 \text { Model Space }\end{array}$} & \multicolumn{2}{|c|}{ Dressed/ERPA } & \multicolumn{2}{|c|}{$\begin{array}{c}\text { Dressed/ERPA } \\
\text { No time inversion }\end{array}$} & \multicolumn{2}{|c|}{$\begin{array}{c}\text { Dressed/ERPA } \\
\rho=5 \text { Model Space }\end{array}$} \\
\hline & $\varepsilon_{n_{\pi}}$ & $Z_{n_{\pi}}$ & $\varepsilon_{n_{\pi}}$ & $Z_{n_{\pi}}$ & $\varepsilon_{n_{\pi}}$ & $Z_{n_{\pi}}$ & $\varepsilon_{n_{\pi}}$ & $Z_{n_{\pi}}$ & $\varepsilon_{n_{\pi}}$ & $Z_{n_{\pi}}$ & $\varepsilon_{n_{\pi}}$ & $Z_{n_{\pi}}$ \\
\hline $2^{+}$ & 23.13 & 0.823 & 23.09 & 0.824 & & & 22.96 & 0.341 & 22.96 & 0.342 & 22.39 & 0.210 \\
\hline $2^{+}$ & & & & & 19.25 & 0.441 & 21.44 & 0.163 & 21.43 & 0.161 & 21.35 & 0.122 \\
\hline $2^{+}$ & & & & & 17.13 & 0.471 & 20.42 & 0.255 & 20.41 & 0.258 & 20.30 & 0.281 \\
\hline $0^{+}$ & 14.28 & 0.010 & 14.28 & 0.010 & 12.85 & 0.232 & 12.15 & 0.001 & 12.15 & 0.001 & 12.06 & 0.005 \\
\hline $2^{+}$ & 13.91 & 0.041 & 13.91 & 0.041 & 13.88 & 0.101 & 12.12 & 0.008 & 12.12 & 0.008 & 12.05 & 0.013 \\
\hline $4^{+}$ & 13.87 & 0.028 & 13.87 & 0.028 & 11.81 & 0.036 & 12.14 & 0.007 & 12.14 & 0.007 & 12.09 & 0.009 \\
\hline $0^{+}$ & & & & & & & 11.28 & 0.092 & 11.28 & 0.092 & 10.97 & 0.184 \\
\hline $1^{-}$ & 9.20 & 1.075 & 9.03 & 1.024 & 9.21 & 1.260 & 10.90 & 0.680 & 10.89 & 0.680 & 10.64 & 0.723 \\
\hline $3^{-}$ & 6.55 & 1.269 & 6.54 & 1.266 & 4.68 & 1.581 & 9.23 & 0.735 & 9.22 & 0.735 & 8.91 & 0.762 \\
\hline $0^{+}$ & 6.52 & 1.820 & 6.36 & 1.596 & 6.74 & 3.055 & & & & & & \\
\hline
\end{tabular}

contribution of $H^{>,<}$and $H^{<,>}$can, in principle, carry information on the Pauli breaking at the level of $3 p-3 h$ and beyond. Therefore, they may become more important for the case of three-phonon calculations. Nevertheless, Table III suggests that they are not likely to play an important role in the description of the spectrum of ${ }^{16} \mathrm{O}$.

\section{Stability vs number of two-phonon configurations}

Figure 7 shows the results for selected solutions of the ERPA equations, obtained by employing different sets of two-phonon states. For any given point, only those configu-

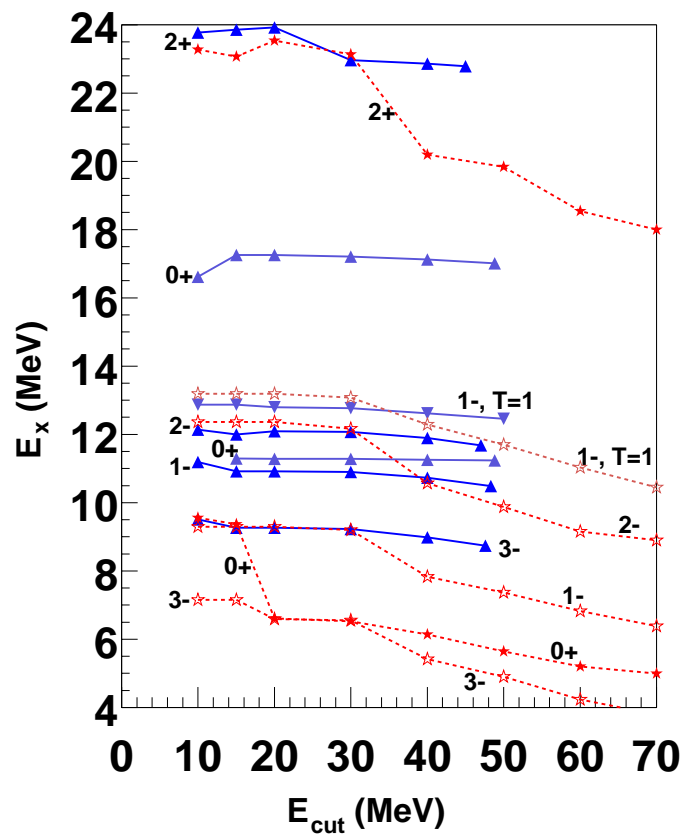

FIG. 7. Dependence of the ERPA solutions on the number of two-phonon states considered. For any given point, all the configuration with energy $\varepsilon_{n_{a}}^{\pi}+\varepsilon_{n_{b}}^{\pi} \leqslant E_{c u t}$ have been included in the calculation. Solid (dashed) lines refer to the results obtained from a dressed (IPM) input propagator. ration with energy $\varepsilon_{n_{a}}^{\pi}+\varepsilon_{n_{b}}^{\pi} \leqslant E_{c u t}$ have been included in the calculation. For $E_{c u t}=70 \mathrm{MeV}$, about 700-1000 twophonon contributions have been included, depending on the channel. This is one order of magnitude larger than the number of $p$ - $h$ states that enter the calculation. Since no twophonon configuration has energy lower than $10 \mathrm{MeV}$, the leftmost points in Fig. 7 correspond to the simple (D)RPA calculation. As the lowest few two-phonon contributions are included, the solutions for these levels show a sudden jump for all the lowest excited states. After this, the results obtained by using an IPM input still continue to exhibit a dependence on the value of the cutoff, roughly lowering states by $1 \mathrm{MeV}$ every time that $E_{\text {cut }}$ increases by $20 \mathrm{MeV}$. In particular, the $0^{+}$and $3^{-}$solutions decrease progressively, eventually heading to RPA instability. This confirms that the values reported in Table I for these states do not correspond to converged results. The situation is much better for the case of a dressed input propagator, for which the low-lying solutions are approximately stable for $E_{c u t}>20 \mathrm{MeV}$. This confirms that corresponding higher-energy two-phonon excitations do not have strong influence as their effects have already been included by the dressing of the sp motion.

In Fig. 7 we also show the results for the most relevant of the $2^{+}$solutions that represent the resonance at about 20 $\mathrm{MeV}$. Here the variation is more appreciable, in particular, for values of the cutoff $E_{c u t}$ comparable with the energy of the state itself. Also, here a more stable behavior is obtained for the case of a dressed input propagator. We conclude that when the nuclear fragmentation is accounted for the choice of $E_{\text {cut }}=30 \mathrm{MeV}$ adopted in Sec. III is adequate for considering the low-lying spectrum.

\section{Size of the model space}

As a second possible source of uncertainty, one may consider the size of the employed model space. The choice of the model space employed in Sec. III allows to take into account excitations up to the $2 p 1 f$ shells. This means that $p$ - $h$ configurations up to $2 \hbar \omega \sim 27 \mathrm{MeV}$ are included, con- 
sistently with the choice for truncating two-phonon states discussed above. The calculations of Refs. [15,39] also show that this is adequate, and suggest that better convergence is to be expected for a calculation with a dressed input. To check this for the present calculation, we have augmented the model space by adding two more h.o. shells, up to $\rho$ $=2 n+\ell=5$, and recomputed the $G$-matrix elements accordingly. The input sp propagator used in the calculations was obtained by including an extra particle pole for every subshell added to both the IPM's Slater determinant and the fragmented dressed propagator. The sp energies for the new shells were chosen by solving the Brueckner-Hartree-Fock equation within the new model space, in an approach analogous to the one of Ref. [6].

The results are compared to the ones obtained for the smaller space in Table III. For the IPM, a sizable variation of the $3^{-}$excitation energy is found. Also, the high values of the $p$ - $h$ strength $Z_{n_{\pi}}$ for both the $0^{+}$and $3^{-}$solutions indicate that these states are approaching instability. As a consequence of the different $3^{-}$excitation energy, which also enters the two-phonon calculation, most of the ERPA solutions are shifted down in energy. The main conclusions of Sec. III, however, remain unchanged. When dressed sp propagators are employed, no dramatic change occurs for the low-lying solutions, and only some states with dominant $p$ - $h$ character are shifted by no more than $1 \mathrm{MeV}$. This suggests that the main conclusions of Sec. III are also not affected by the truncation of the model space, whence the bulk of the missing correlations has to be looked for in an extension of the diagrammatic expansion. We note, however, that a more attractive $G$ matrix is expected to be obtained if the continuum outside the model space were modified by accounting for self-energy corrections. This feature may lead to a further improvement of the present results. In addition, one may explore the inclusion of effective three-body force as due to propagation outside the model space. Such effective forces are critical in obtaining the correct binding energy in no-core shell model calculations reported in Ref. [31].

\section{CONCLUSIONS}

The dressed RPA equations have been extended to account for the coupling of two $p$ - $h$ phonons in forming the excited states of a many-body system. The coupling among $p-h$ and multiphonon configurations is conceptually similar to the interacting boson model of Ref. [25], but limited (in this application) to only two-phonon admixtures. Nevertheless, the present approach has the added advantage of taking into account both the effects of nuclear fragmentation and the RPA-like correlations, as generated by two-phonon fluctuations in the ground state.

The resulting extended RPA formalism has been applied to study the excitation spectrum of ${ }^{16} \mathrm{O}$. The results suggest a sizable mixing of $p$ - $h$ and two-phonon configurations for the case of the $2^{+}$quadrupole resonance at $20.7 \mathrm{MeV}$. Other solutions, carrying quantum numbers that cannot be generated by the simple DRPA, are obtained with this method, among which are a few isoscalar negative-parity states and a triplet with $J^{\pi}=0^{+}, 2^{+}$, and $4^{+}$near $12 \mathrm{MeV}$, in accord with experiment. In particular, the states in the triplet were seen to be almost pure two-phonon configurations obtained by coupling two $3^{-}$phonons. This feature confirms the $2 p-2 h$ character of these states. The results are less satisfying for the low-lying positive-parity states, which are known to require a proper description of $4 p-4 h$ excitations. The present approach predicts the lowest solution for the isoscalar $0^{+}$channel to be at $11.3 \mathrm{MeV}$, considerably above the first experimental excited state. A sizable component of this solution is seen to be generated by the coupling of two of the lowest $0^{+}$phonons themselves. This suggests that important contribution of $4 p-4 h$ excitation may be included already at the two-phonon level in a self-consistent fashion. Other correlations beyond the $2 p-2 h$ level are partially included in the present calculation through the dressing of the propagators and the RPA approach. As expected, though, the present implementation is not sufficient to obtain a complete description of the low-lying excitation spectrum of ${ }^{16} \mathrm{O}$. The results obtained in this work show that an interaction between multiphonon configurations is still missing. This can be achieved by including $p-p$ and $h$ - $h$ correlations between different $p$ - $h$ phonons. Also, the inclusion of up to four-phonon states is expected to be relevant for this system.

Finally, the stability of the present results has been tested with respect to the truncation of the model space and to the number of two-phonon configurations accounted for. It was found that the effects of nuclear fragmentation acts to "renormalize" the sp propagator, making the solutions of the ERPA fairly independent of higher energy configurations. As discussed in Sec. IV, this feature generates stable solutions with respect to the size of the model space and the corresponding $G$-matrix interaction used in this work.

The four-phonon calculation of Refs. $[25,26]$ give a very good description of the excitation spectrum of ${ }^{16} \mathrm{O}$. Those findings and the effects of fragmentation discussed in this paper suggest that the present ERPA formalism can be suitably extended to generate a satisfactory description of this nucleus, within the framework of SCGF. Such extensions are presently under consideration. Such a calculation may also significantly reduce the discrepancy between the measured and the theoretical sp spectral function for this nucleus [9].

\section{ACKNOWLEDGMENTS}

This work was supported in part by the U.S. National Science Foundation under Grant Nos. PHY-9900713 and PHY-0140316, and in part by the Natural Sciences and Engineering Research Council of Canada (NSERC).

\section{APPENDIX: EXTENDED DRPA EQUATION WITH TWO-PHONON CONTRIBUTIONS}

When reducing the kernel of the Bethe-Salpeter equation (3) to a two-time quantity, one has to deal with the fact that some lines in the diagrammatic expansion continue to propagate unperturbed while some interaction occurs between other particles. This is also the case for the diagrams of Figs. 1 and 4. This situation can be overcome by redefining the objects that appear in Eq. (9) and promoting the quantum 
numbers $\{n, k\}$ - which label particle and hole fragments- to external indices. The usual form of the $p-h$ propagator, Eq. (1), is obtained only as a last step of the calculation by performing the sum over the $\{n, k\}$ indices [39,28].

The separation of the propagators $\Pi^{f}(\omega)$ and $\Pi(\omega)$ into forward and backward components, outlined in Sec. II B, is a natural consequence of adopting this prescription. Indeed, particle and hole external lines turn into each other by time inversion and become quantities that depend on different quantum numbers, i.e., the fragmentation indices $\{n, k\}$. For this reason, Eqs. (6) and (7) are only formal relations and the arrow can be substituted by an equal sign only before summing over all the particle and hole fragments. The free polarization propagator (5) naturally splits in two components that are purely forward going and backward going. The relevant Lehmann representations are

$$
\begin{aligned}
\Pi_{\alpha n_{\alpha} \beta k_{\beta}, \gamma n_{\gamma} \delta k_{\delta}}^{f>}(\omega) & =\delta_{n_{\alpha}, n_{\gamma}} \delta_{k_{\beta}, k_{\delta}} \frac{\left(\mathcal{X}_{\alpha}^{n_{\alpha}} \mathcal{Y}_{\beta}^{k_{\beta}}\right) \mathcal{X}_{\gamma}^{n_{\alpha}} \mathcal{Y}_{\delta}^{k_{\beta}}}{\omega-\left(\varepsilon_{n_{\alpha}}^{+}-\varepsilon_{k_{\beta}}^{-}\right)+i \eta} \\
& \equiv G^{\dagger} \frac{1}{\omega-D+i \eta} G, \\
\Pi_{\alpha k_{\alpha} \beta n_{\beta}, \gamma k_{\gamma} \delta n_{\delta}}^{f<}(\omega) & =-\delta_{k_{\alpha}, k_{\gamma}} \delta_{n_{\beta}, n_{\delta}} \frac{\mathcal{Y}_{\alpha}^{k_{\alpha}} \mathcal{X}_{\beta}^{n_{\beta}}\left(\mathcal{Y}_{\gamma}^{k_{\alpha}} \mathcal{X}_{\delta}^{n_{\beta}}\right)^{*}}{\omega+\left(\varepsilon_{n_{\beta}}^{+}-\varepsilon_{k_{\alpha}}^{-}\right)-i \eta} \\
& \equiv\left(G^{*}\right)^{\dagger} \frac{-1}{\omega+D-i \eta} G^{*},
\end{aligned}
$$

where no summation is implied, and a shorter notation for the unperturbed poles and residues has been introduced. The quantities $D$ and $G$ can be thought as matrices whose elements contain all the unperturbed poles and residues, respectively. Note that in this case $D$ is diagonal and depends on the fragmentation indices $\{n, k\}$ only, while $G$ is rectangular because its column indices depend on the model space orbitals $\{\alpha\}$ as well.

The separation of the complete propagator is a little more complicated. The splitting of Eqs. (7) and (8) involves the time direction of the outgoing lines only. Since the RPA series contains contributions that can invert several times the sense of propagation of the $p$ - $h$ diagrams, both forward and backward poles can appear in each component,

$$
\begin{aligned}
& \Pi_{\alpha n_{\alpha} \beta k_{\beta}, \gamma \delta}^{>}(\omega)=\sum_{n \neq 0} \frac{\left(\mathcal{Z}_{\alpha n_{\alpha} \beta k_{\beta}}^{>n}\right) * \mathcal{Z}_{\gamma \delta}^{n}}{\omega-\varepsilon_{n}^{\pi}+i \eta} \\
& -\sum_{n \neq 0} \frac{\mathcal{Z}_{\beta k_{\beta}{ }^{\alpha n_{\alpha}}}^{<{ }_{2}}\left(\mathcal{Z}_{\delta \gamma}^{n}\right)^{*}}{\omega+\varepsilon_{n}^{\pi}-i \eta} \\
& \Pi_{\alpha k_{\alpha} \beta n_{\beta}, \gamma \delta}^{<}(\omega)=\sum_{n \neq 0} \frac{\left(\mathcal{Z}_{\alpha k_{\alpha} \beta n_{\beta}}^{<n}\right) * \mathcal{Z}_{\gamma \delta}^{n}}{\omega-\varepsilon_{n}^{\pi}+i \eta} \\
& -\sum_{n \neq 0} \frac{\mathcal{Z}_{\beta n_{\beta}{ }^{\alpha k} k_{\alpha}}^{>}\left(\mathcal{Z}_{\delta \gamma}^{n}\right)^{*}}{\omega+\varepsilon_{n}^{\pi}-i \eta}
\end{aligned}
$$

In Eqs. (A3) and (A4) the spectroscopic amplitude splits in two contributions $\mathcal{Z}^{>}$and $\mathcal{Z}^{<}$. These appear unchanged in both equations due to the time-inversion symmetries obeyed by the $p$ - $h$ states. In terms of these definitions, and applying the summation prescription, Eq. (7) can be exactly formulated as follows:

$$
\begin{gathered}
\mathcal{Z}_{\alpha \beta}^{n}=\sum_{n_{1}, k_{2}}\left[\mathcal{Z}_{\alpha n_{1} \beta k_{2}}^{>n}+\mathcal{Z}_{\alpha k_{2} \beta n_{1}}^{<n}\right], \\
\Pi_{\alpha \beta, \gamma \delta}^{>}(\omega)=\sum_{n_{1}, k_{2}}\left[\Pi_{\alpha n_{1} \beta k_{2}, \gamma \delta}^{>}(\omega)+\Pi_{\alpha k_{2} \beta n_{1}, \gamma \delta}^{<}(\omega)\right] .
\end{gathered}
$$

Finally, the contributions of two-phonon diagrams in the forward and backward directions can be expressed in Lehmann representation as well,

$$
\begin{aligned}
W_{\alpha n_{\alpha} \beta k_{\beta}, \gamma n_{\gamma} \delta k_{\delta}}^{>}(\omega) & =\sum_{n_{a}, n_{b}} \frac{\left(K_{\alpha n_{\alpha} \beta k_{\beta}}^{>n_{a} n_{b}}\right) * K_{\gamma n_{\gamma} \delta k_{\delta}}^{>n_{a} n_{b}}}{\omega-\left(\varepsilon_{n_{a}}^{\pi}+\varepsilon_{n_{b}}^{\pi}\right)+i \eta} \\
& =K^{>\dagger} \frac{1}{\omega-E+i \eta} K^{>}, \\
W_{\alpha k_{\alpha} \beta n_{\beta}, \gamma k_{\gamma} \delta n_{\delta}}^{<}(\omega) & =\sum_{n_{a}, n_{b}}-\frac{K_{\alpha k_{\alpha} \beta n_{\beta}}^{<n_{a} n_{b}}\left(K_{\gamma k_{\gamma} \delta n_{\delta}}^{<n_{n} n_{b}}\right) *}{\omega+\left(\varepsilon_{n_{a}}^{\pi}+\varepsilon_{n_{b}}^{\pi}\right)-i \eta} \\
& =K^{<\dagger} \frac{-1}{\omega-E-i \eta} K^{<,},
\end{aligned}
$$

where $\varepsilon_{n_{a}}^{\pi}$ and $\varepsilon_{n_{b}}^{\pi}$ are the energies of the intermediate $p-h$ phonons.

\section{ERPA matrix}

The eigenvalue equation for the ERPA is obtained in the usual way, by substituting Eqs. (A1)-(A4) into Eq. (9) and then extracting the residues of the $p$ - $h$ poles $\varepsilon_{n}^{\pi}$. The result is an eigenvalue equation in terms of the vectors $\mathcal{Z}^{>}$and $\mathcal{Z}^{<}$.

To linearize the problem, we introduce the following components:

$$
\begin{aligned}
& X_{n_{\alpha} k^{\prime}}^{(1)} \equiv \frac{1}{\omega-D+i \eta} G\left\{\left[V+W^{>}(\omega)\right]\left(\mathcal{Z}^{>}\right)^{*}\right. \\
&\left.-\left(V+H^{>,<}\right)\left(\mathcal{Z}^{<}\right)^{*}\right\}, \\
& Y_{k_{\alpha} n_{\beta}}^{(1)} \equiv \frac{1}{\omega+D-i \eta} G^{*}\left\{\left(V+H^{<,>}\right)\left(\mathcal{Z}^{>}\right)^{*}\right. \\
&\left.-\left[V+W^{<}(\omega)\right]\left(\mathcal{Z}^{<}\right)^{*}\right\}, \\
& X_{n_{a} n_{b}}^{(2)} \equiv \frac{1}{\omega-E+i \eta} K^{>}\left(\mathcal{Z}^{>}\right)^{*},
\end{aligned}
$$




$$
Y_{n_{a} n_{b}}^{(2)} \equiv \frac{1}{\omega+E-i \eta} K^{<}\left(\mathcal{Z}^{<}\right) *
$$

where $X^{(1)}$ and $Y^{(1)}$ represent the $p$ - $h$ amplitudes that appear in the standard (D)RPA equations $[47,16]$, and $X^{(2)}$ and $Y^{(2)}$ are the analogous two-phonon amplitudes introduced by the ERPA approach. The components $X^{(1)}$ and $Y^{(1)}$ are related to $\mathcal{Z}^{>}$and $\mathcal{Z}^{<}$, respectively, by

$$
\left(\mathcal{Z}_{\alpha n_{\alpha} \beta k_{\beta}}^{>}\right)^{*}=\mathcal{X}_{\alpha}^{n} \mathcal{Y}_{\beta}^{k_{\beta}} X_{n_{\alpha} k_{\beta}}^{(1)}=G^{\dagger} X^{(1)}
$$

$$
\mathbf{M}=\left[\begin{array}{cc}
G V G^{\dagger}+D & G K^{>\dagger} \\
K^{>} G^{\dagger} & E \\
-G^{*}\left[V+H^{<,>}\right] G^{\dagger} &
\end{array}\right.
$$

The off-diagonal $2 \times 2$ blocks in Eq. (A12) describe diagrams in which the time direction of propagation is inverted. In the present case the only nonvanishing elements are the ones that involve the inversion of a single $p-h$ state into a $h-p$ one, or vice versa. These correspond to the sum of the first-order term $V$, which represents the kernel of the bare RPA, and the more complex diagrams of Fig. 4. Blank spaces would, in principle, allow to include more complicated contributions that involve time inversion of two-phonon diagrams into $p$ - $h$ or $h-p$ configurations. These contributions are not expected to play a relevant role for the present problem. It must be noted that if the terms involving the matrix $G K^{\dagger}$ are discarded in Eq. (A12), the components $X^{(1)}$ and $Y^{(1)}$ decouple from $X^{(2)}$ and $Y^{(2)}$. In this case, Eqs. (9) reduce to the $p$ - $h$-DRPA one (4) and matrix (A12) would take the form of the standard RPA matrix [47]. The normalization condition is derived in the usual way, by extracting the contribution of order zero of the expansion around a given pole and by employing the conjugate equation. One eventually obtains [16]

$$
\sum_{i=1,2}\left(X^{(i) \dagger} X^{(i)}-Y^{(i) \dagger} Y^{(i)}\right)=1,
$$

where the inner product of the vectors $X^{(i)}$ and $Y^{(i)}$ is implied.

\section{Matrix elements for $p$ - $h$ ERPA}

In the following, we give the explicit expression for the matrix elements of Eq. (A12). The contributions originating from the standard (D)RPA equation are (here and below, summations over repeated greek indices are understood)

$$
\begin{gathered}
\left(G V G^{\dagger}\right)_{n_{1} k_{2}, n_{3} k_{4}}=\mathcal{X}_{\alpha}^{n_{1}} \mathcal{Y}_{\beta}^{k_{2}} V_{\alpha \nu, \beta \mu}\left(\mathcal{X}_{\mu}^{n_{3}} \mathcal{Y}_{\nu}^{k_{4}}\right)^{*}, \\
{\left[G^{*} V\left(G^{*}\right)^{\dagger}\right]_{k_{1} n_{2}, k_{3} n_{4}}=\left(\mathcal{Y}_{\alpha}^{k_{1}} \mathcal{X}_{\beta}^{n_{2}}\right)^{*} V_{\alpha \nu, \beta \mu} \mathcal{Y}_{\mu}^{k_{3}} \mathcal{X}_{\nu}^{n_{4}},}
\end{gathered}
$$

$$
\left(\mathcal{Z}_{\alpha k_{\alpha} \beta n_{\beta}}^{<}\right)^{*}=\mathcal{Y}_{\alpha}^{k_{\alpha}} \mathcal{X}_{\beta}^{n_{\beta}} Y_{k_{\alpha} n_{\beta}}^{(1)}=\left(G^{*}\right)^{\dagger} Y^{(1)}
$$

Equations (9) can be put in the form of a linear eigenvalue equation,

$$
\omega\left(\begin{array}{l}
X^{(1)} \\
X^{(2)} \\
Y^{(1)} \\
Y^{(2)}
\end{array}\right)=\mathbf{M}\left(\begin{array}{l}
X^{(1)} \\
X^{(2)} \\
Y^{(1)} \\
Y^{(2)}
\end{array}\right)
$$

where the matrix $\mathbf{M}$ is defined as

$$
\begin{gathered}
\left(G^{*} V G^{\dagger}\right)_{k_{1} n_{2}, n_{3} k_{4}}=\left(\mathcal{Y}_{\alpha}^{k_{1}} \mathcal{X}_{\beta}^{n_{2}}\right)^{*} V_{\alpha \nu, \beta \mu}\left(\mathcal{X}_{\mu}^{n_{3}} \mathcal{Y}_{\nu}^{k_{4}}\right)^{*}, \\
{\left[G V\left(G^{*}\right)^{\dagger}\right]_{n_{1} k_{2}, k_{3} n_{4}}=\mathcal{X}_{\alpha}^{n_{1}} \mathcal{Y}_{\beta}^{k_{2}} V_{\alpha \nu, \beta \mu} \mathcal{Y}_{\mu}^{k_{3}} \mathcal{X}_{\nu}^{n_{4}},}
\end{gathered}
$$

with the corresponding unperturbed $p$ - $h$ energies,

$$
D_{n_{1} k_{2}}=\operatorname{diag}\left\{\varepsilon_{n_{1}}^{+}-\varepsilon_{k_{2}}^{-}\right\}
$$

The interaction between two-phonon intermediate states and the $p$ - $h$ ones is given by

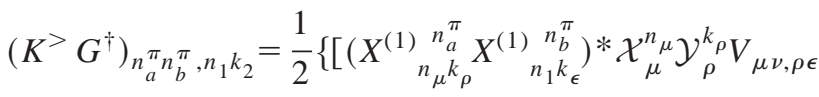

$$
\begin{aligned}
& \times \mathcal{Y}_{\epsilon}^{k} \epsilon\left(\mathcal{Y}_{\nu}^{k_{2}}\right) *-\left(X^{(1)}{ }_{n_{\mu} k_{\rho}^{k}}^{n_{a}^{\pi}} X^{(1)}{ }_{n_{\nu} k_{2}}^{n_{b}^{\pi}}\right) * \mathcal{X}_{\mu}^{n_{\mu}} \\
& \left.\left.\times \mathcal{Y}_{\rho}^{k_{\rho}} V_{\mu \nu, \rho \epsilon}\left(\mathcal{X}_{\epsilon}^{n_{1}}\right) * \mathcal{X}_{\nu}^{n_{\nu}}\right]+\left[n_{a}^{\pi} \leftrightarrow n_{b}^{\pi}\right]\right\},
\end{aligned}
$$

where $n_{a}^{\pi}$ and $n_{b}^{\pi}$ are the quantum numbers of the two phonons that form the intermediate state. The quantities $X^{(1)}{ }_{n_{1} k_{2}}^{k^{\pi}}$ are the forward-going amplitudes of the intermediate phonons (A9) or (A10) and are obtained from the previous solution of the polarization propagator. Note that $K^{>} G^{\dagger}$ is symmetric under the exchange of the two indices $n_{a}^{\pi}$ and $n_{b}^{\pi}$, as required by the boson character of the $p-h$ phonons. The 
factor $\frac{1}{2}$ assures that no double counting happens when only two free propagators $\Pi^{f}(\omega)$ are coupled. Due to the timeinversion properties of $n p-n h$ states, the corresponding contribution for backward-going propagation is simply related to the above one:

$$
\left[K^{<}\left(G^{*}\right)^{\dagger}\right]_{n_{a} \pi_{b} n_{b}, k_{1} n_{2}}=\left\{\left(K^{>} G^{\dagger}\right)_{n_{a} \pi_{n} n_{b}, n_{2} k_{1}}\right\}^{*} .
$$

The analytical expression for the contribution of the timeinversion diagrams is a little more complicated. First, we introduce the following quantity that corresponds to the gen- eration of a two-phonon state by a fluctuation of the nuclear mean field,

$$
\begin{aligned}
& U_{n_{a} n_{b} \pi}=\frac{1}{-\left(\varepsilon_{n_{a}}^{\pi}+\varepsilon_{n_{b}}^{\pi}\right)}\left[\left(\mathcal{Y}_{\sigma}^{k_{\sigma}} \mathcal{Y}_{\lambda}^{k_{\lambda}}\right)^{*} V_{\sigma \lambda, \mu \nu}\right.
\end{aligned}
$$

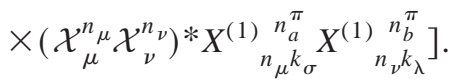

This is also symmetric under the exchange of the indices $n_{a}^{\pi}$ and $n_{b}^{\pi}$. Then, the matrix elements containing the timeinversion diagrams, $H^{>,}<$and $H^{<,>}$, can be written as follows (a summation over $n_{a}^{\pi}$ and $n_{b}^{\pi}$ is also implied):

$$
\begin{aligned}
& {\left[G H^{>,<}\left(G^{*}\right)^{\dagger}\right]_{n_{1} k_{2}, k_{3} n_{4}}=\left\{\left[\left(G^{*}\right) H^{<,>} G^{\dagger}\right]_{k_{3} n_{4}, n_{1} k_{2}}\right\}^{*}} \\
& =\frac{1}{2}\left\{\left[\mathcal{X}_{\alpha}^{n_{1}} \mathcal{Y}_{\beta}^{k_{2}} V_{\alpha \epsilon, \beta \rho}\left(\mathcal{X}_{\rho}^{n_{\rho}} \mathcal{Y}_{\epsilon}^{k}\right)^{*} X^{(1)}{ }_{n_{\rho^{k}} n_{a}^{\pi}}^{\pi} X_{n_{4} k_{\epsilon}}^{(1)}{ }_{n_{b}^{\pi}}^{\pi}\right]+\left[-\left(n_{1} \leftrightarrow n_{4}\right)-\left(k_{2} \leftrightarrow k_{3}\right)+\left(n_{1} \leftrightarrow n_{4}, k_{2} \leftrightarrow k_{3}\right)\right]\right.
\end{aligned}
$$

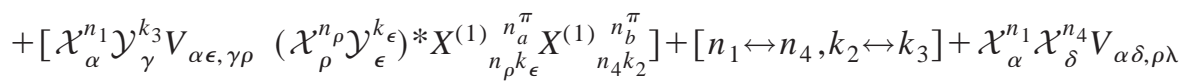

$$
\begin{aligned}
& \left.\times\left(\mathcal{X}_{\rho}^{n_{\rho}} \mathcal{X}_{\lambda}^{n_{\lambda}}\right) * X^{(1)}{ }_{n_{\rho} k_{2}}^{n_{a}^{\pi}} X^{(1)}{ }_{n_{\lambda} k_{3}}^{n_{b}^{\pi}}+\mathcal{Y}_{\rho}^{k_{\rho}} \mathcal{Y}_{\lambda}^{k_{\lambda}} V_{\rho \lambda, \beta \gamma}\left(\mathcal{Y}_{\beta}^{k_{2}} \mathcal{Y}_{\gamma}^{k_{3}}\right)^{*} X^{(1)}{ }_{n_{1} k_{\rho}}^{n_{a}^{\pi}} X^{(1)}{ }_{n_{4} k_{\lambda}}^{n_{b}^{\pi}}\right\}\left(U_{n_{a} n_{b} \pi}\right)^{*}
\end{aligned}
$$

Finally, the two-phonon unperturbed energies are given by

$$
E_{n_{a} n_{b}^{\pi}}=\operatorname{diag}\left\{\varepsilon_{n_{a}}^{\pi}+\varepsilon_{n_{b}}^{\pi}\right\} .
$$

[1] M. Leuschner et al., Phys. Rev. C 49, 955 (1994).

[2] H. Müther and W.H. Dickhoff, Phys. Rev. C 49, R17 (1994).

[3] M. Radici, S. Boffi, S.C. Pieper, and V.R. Pandharipande, Phys. Rev. C 50, 3010 (1994).

[4] A. Polls, H. Müther, and W.H. Dickhoff, Phys. Rev. C 51, 3040 (1995).

[5] K. Amir-Azimi-Nili, H. Müther, L.D. Skouras, and A. Polls, Nucl. Phys. A604, 245 (1996).

[6] W.J.W. Geurts, K. Allaart, W.H. Dickhoff, and H. Müther, Phys. Rev. C 53, 2207 (1996).

[7] D. Van Neck, M. Waroquier, A.E.L. Dieperink, S.C. Pieper, and V.R. Pandharipande, Phys. Rev. C 57, 2308 (1998).

[8] A. Fabrocini and G. Co', Phys. Rev. C 63, 044319 (2001).

[9] C. Barbieri and W.H. Dickhoff, Phys. Rev. C 65, 064313 (2002)

[10] Kh. Gad and H. Müther, Phys. Rev. C 66, 044301 (2002).

[11] J.M. Udías, J.A. Caballero, E. Moya de Guerra, J.R. Vignote, and A. Escuderos, Phys. Rev. C 64, 024614 (2001).

[12] M.K. Gaidarov et al., Phys. Rev. C 61, 014306 (2000).

[13] M. Radici, W.H. Dickhoff, and E. Roth Stoddard, Phys. Rev. C 66, 014613 (2002).

[14] P. Czerski, W.H. Dickhoff, A. Faessler, and H. Müther, Phys. Rev. C 33, 1753 (1986).

[15] M.G.E. Brand, K. Allaart, and W.H. Dickhoff, Phys. Lett. B 214, 483 (1988)

[16] M.G.E. Brand, K. Allaart, and W.H. Dickhoff, Nucl. Phys. A509, 1 (1990)
[17] G.F. Bertsch and S.F. Tsai, Phys. Rep. 18, 125 (1975); R. Id Betan, R.J. Liotta, N. Sandulescu, and T. Verste, Phys. Rev. Lett. 89, 042501 (2002); N. Michel, W. Nazarewicz, M. Płoszajczak, and K. Bennaceur, ibid.. 89, 042502 (2002)

[18] J. Yuan, Ph.D. thesis, Washington University, 1994.

[19] A.P. Zucker, B. Buck, and J.B. McGrory, Phys. Rev. Lett. 21, 39 (1968).

[20] B.S. Reehal and B.H. Wildenthal, Part. Nuclei 6, 137 (1973).

[21] W.C. Haxton and C.J. Johnson, Phys. Rev. Lett. 65, 1325 (1990).

[22] E.K. Warburton, B.A. Brown, and D.J. Millener, Phys. Lett. B 293, 7 (1992).

[23] T.N. Buti et al., Phys. Rev. C 33, 755 (1986).

[24] G.E. Brown and A.M. Green, Nucl. Phys. 75, 401 (1966).

[25] H. Feshbach and F. Iachello, Phys. Lett. 45B, 7 (1973).

[26] H. Feshbach and F. Iachello, Ann. Phys. (N.Y.) 84, 211 (1974).

[27] W. H. Dickhoff, in Condensed Matter Theories, edited by J. S. Arponen, R. F. Bishop, and M. Manninen (Plenum, New York, 1988), Vol. 3, p. 261.

[28] C. Barbieri and W.H. Dickhoff, Phys. Rev. C 63, 034313 (2001).

[29] B.S. Pudliner, V.R. Pandharipande, J. Carlson, S.C. Pieper, and R.B. Wiringa, Phys. Rev. C 56, 1720 (1997); S.C. Pieper, K. Varga, and R.B. Wiringa, ibid. 66, 044310 (2002).

[30] P. Navrátil, J.P. Vary, and B.R. Barrett, Phys. Rev. C 62, 054311 (2000); P. Navrátil and B.R. Barrett, ibid. 57, 3119 (1998). 
[31] P. Navrátil and W.E. Ormand, Phys. Rev. Lett. 88, 152502 (2002).

[32] G. Baym and L.P. Kadanoff, Phys. Rev. 124, 287 (1961).

[33] G. Baym, Phys. Rev. 127, 1391 (1962).

[34] L. P. Kadanoff and G. Baym, Quantum Statistical Mechanics (Benjamin, New York, 1962).

[35] H. Lehmann, Nuovo Cimento 11, 342 (1952).

[36] A. L. Fetter and J. D. Walecka, Quantum Theory of ManyParticle Physics (McGraw-Hill, New York, 1971).

[37] A. A. Abrikosov, L. P. Gorkov, and I. E. Dzyaloshinski, Methods of Quantum Field Theory in Statistical Physics (Dover, New York, 1975).

[38] C. Barbieri, Ph.D. thesis, Washington University, 2002.

[39] W.J.W. Geurts, K. Allaart, and W.H. Dickhoff, Phys. Rev. C 50, 514 (1994).

[40] W. Hengeveld, W.H. Dickhoff, and K. Allaart, Nucl. Phys.
A451, 269 (1986).

[41] C. Barbieri and W. H. Dickhoff, in Proceedings of the Fifth Workshop on Electromagnetically Induced Two-hadron Emission, edited by P. Grabmayr et al. (University of Lund, Lund, 2001), p. 108.

[42] R. Machleidt, Adv. Nucl. Phys. 19, 191 (1989).

[43] H. Müther and P. Sauer, in Computational Nuclear Physics, edited by K.-H. Langanke et al. (Springer, Berlin, 1993).

[44] F. Ajzenberg-Selove, Nucl. Phys. A375, 1 (1982).

[45] M.S. Zisman, E.A. McClatchie, and B.G. Harvey, Phys. Rev. C 2, 1271 (1970); J. Lowe and A.R. Barnett, Nucl. Phys. A187, 323 (1972).

[46] T. Berggren, Nucl. Phys. A109, 265 (1968); A389, 261 (1982).

[47] P. Ring and P. Schuck, The Nuclear Many-body Problem (Springer, New York, 1980). 